\title{
Un micete patogeno per l'uomo e per le piante
}

per

O. Verona.

(Dall'Istituto di Microbiologia, Facoltà di Agraria, R. Università di Firenze)

(29.II.1940.)

Nel 1929 L. Maffer descriveva una nuova specie di Cephalosporium (C. Serrae Maffei) causa di cheratomicosi nell'Uomo.

Il caso venne riscontrato dal Dr. G. M. Serra nella Clinica Oculistica di Parma. Più esattamente il micete si sviluppò in seguito a penetrazione di corpo estraneo nell'occhio di un contadino mentre stava scaricando del fieno.

Lo sviluppo del fungo dette luogo ad un focolaio micotico che, osservato col microscopio corneale di ZEISs-CzAPSKI, si presentava come una massa bianca di aspetto ceroso, rilevata sulla cornea a forma di mandorla, presentante un contorno sinuoso ed irregolare. Lo studio morfocolturale del fungo non consentì il riferimento a specie note del genere Cephalosporium onde il micete fu tenuto distinto come specie nuova.

In epoca del tutto recente VAN BEYMa rivedendo dal punto di vista sistematico alcuni Verticillium del gruppo „dahliae" prende occasione per riportare Cephalosporium Serrae al genere Verticillium istituendo Verticillium Serrae (Maffei) van Beyma.

Ora, Vert. Serrae, rientra, insieme ad altre specie - incluso Vert. amaranti Ver. et Cecc. da noi isolato da un caso di tracheomicosi dell'Amaranto - nel gruppo di Verticillium dahliae: in un gruppo di miceti, cioè, abbastanza diffuso in Natura e causa di gravi morbi nelle piante superiori.

Siamo in presenza, quindi, di un gruppo di miceti che si manifestano ad un tempo sicuramente parassiti per l'Uomo e per $i$ vegetali.

Il fatto, in difetto di osservazioni del genere, ci è parso meritevole di segnalazione; come appare meritevole di studio l'indagine comparativa, dal punto di vista patogenetico, di altre specie del genere o affini e note parassite di piante.

D'altronde, anche una revisione dei vari Cephalosporium descritti patogeni par l'Uomo è da ritenersi opportuna in vista di un loro eventualmente più esatto riferimento sistematico.

\section{Lavori citati}

Maffei, L., Atti Ist. Bot. R. Univ. Pavia; Ser. IV, 1, 183, (1929) - Van Beyma, F. H., Journ. of Microb. a. Ser. (Netherlands Society of Microb.); 6, 34, (1939-40)-Verona, 0. e Ceccarelli, A., Phytopathol. Zeitschr., 8, 4, (1935). 